\title{
High-Quality Optical Hotspots with Topology-Protected Robustness
}

\author{
Changxu Liu* and Stefan A. Maier* \\ Cite This: ACS Photonics 2022, 9, 241-248 \\ Read Online
}

ABSTRACT: Optical hotspots underpin a wide variety of photonic devices ranging from sensing, nonlinear generation to photocatalysis, taking advantage of the strong light-matter interaction at the vicinity of photonic nanostructures. While plasmonic nanostructures have been widely used for strongly localized electromagnetic energy on surfaces, they suffer from high loss and consequently a low quality factor. Resonance-based dielectric structures provide an alternative solution with a larger quality factor, but there is a mismatch between the maximum values of the light confinement (quality factor) and the leakage (intensity in the near-field). Here, we propose to apply the concept of topological photonics to the formation of hotspots, producing
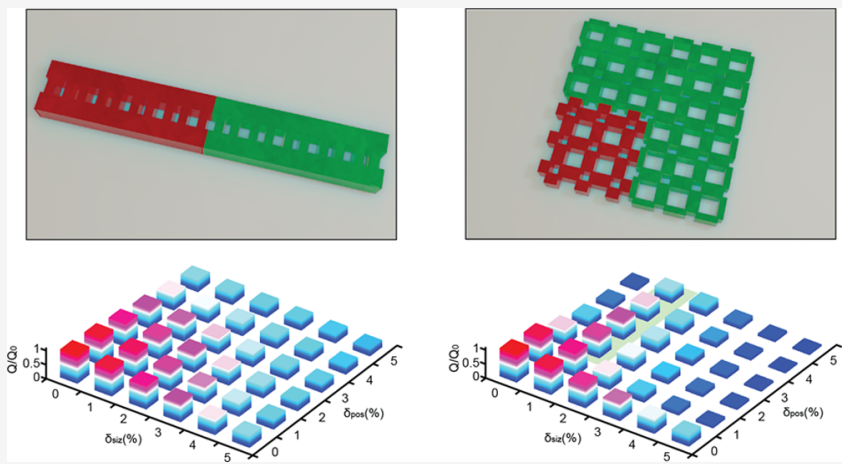
them in both topological edge states and topological corner states. The topology secures strong light localization at the surface of the nanostructures where the underlying topological invariant shows a jump, generating a field hotspot with simultaneous increment of quality factor and light intensity. Meanwhile, it leverages a good robustness to fabrication imperfection including fluctuation in shape and misalignment. After a systematic investigation and comparison of the robustness between $1 \mathrm{D}$ and $2 \mathrm{D}$ topological structures, we conclude that the hotspots from $1 \mathrm{D}$ topological edge states promise a fertile playground for emerging applications that require both enhanced light intensity and high spectral resolution.

KEYWORDS: hotspots, topological photonics, disorder, dielectric resonators

\section{INTRODUCTION}

Optical hotspots with tightly focused electromagnetic fields are underpinning a repertoire of devices requiring a strong interaction between materials and photons. Hotspots with near-field enhancement provide a region with significantly enlarged photon density, facilitating functionalities otherwise demanding significant light intensity. By introducing different types of materials into hotspots, a variety of applications have been achieved, ranging from nonlinear materials for harmonic generation, ${ }^{1,2}$ semiconductors for photovoltaics, ${ }^{3,4}$ chemical or biomolecules for optical sensing based on either Raman scattering, resonance shift or absorption variation, ${ }^{5-15}$ to luminescent materials for enhanced fluorescence $e^{16-20}$ and proper chemicals for photocatalysis. ${ }^{21-24}$ In addition, optical hotspots can even function as optical tweezers for nanoparticle manipulation. ${ }^{25,26}$

Plasmonic nanostructures ${ }^{1,3,4,7,9,16-18,21,26}$ have been one of the most successfully used solutions for hotspot generation, through the excitation of surface plasmon resonances, collective and coherent oscillations of free electrons. ${ }^{27}$ Despite the intense near field enhancement and matured fabrication technologies, the mainstream plasmonic structures composed of metals such as $\mathrm{Au}$ and $\mathrm{Ag}$ suffer from excessive losses at optical frequencies. ${ }^{28}$ Consequently, plasmonic structures exhibit local heating and a low quality factor, limiting the performance in many applications. For example, the heat generated by the nano- antennas may perturb or even malfunction nanoemitters, molecules, and proteins in the vicinity. ${ }^{29-31}$ Meanwhile, a low value of the quality factor corresponds to a wide spectral width in reflection/absorption spectra, reducing the spectral resolution (or sensitivity) of optical sensing based on resonance shift or absorption variation. ${ }^{11,12,15}$

Resonant dielectric nanostructures including photonic crystals, ${ }^{10,11}$ whispering gallery modes, ${ }^{15}$ Mie resonators, ${ }^{32}$ and bound states in the continuum (BIC) $)^{33,34}$ provide an alternative to produce hotspots. A variety of devices have been successfully realized for sensing, fluorescence enhancement, and surfaceenhanced Raman spectroscopy, achieving a high quality factor simultaneously. ${ }^{5,6,10-15,19,20,35}$ However, there is a mismatch between the maximum values of quality factor and near-field enhancement; ${ }^{36}$ the electric field may increase when the quality factor decreases. When the value of quality factor is huge, strong confinement with minimized leakage modes maximizes the quality factor but limits the light intensity around nanostructures. For example, the symmetry of the BIC structures was

Received: September 20, 2021

Published: December 23, 2021 

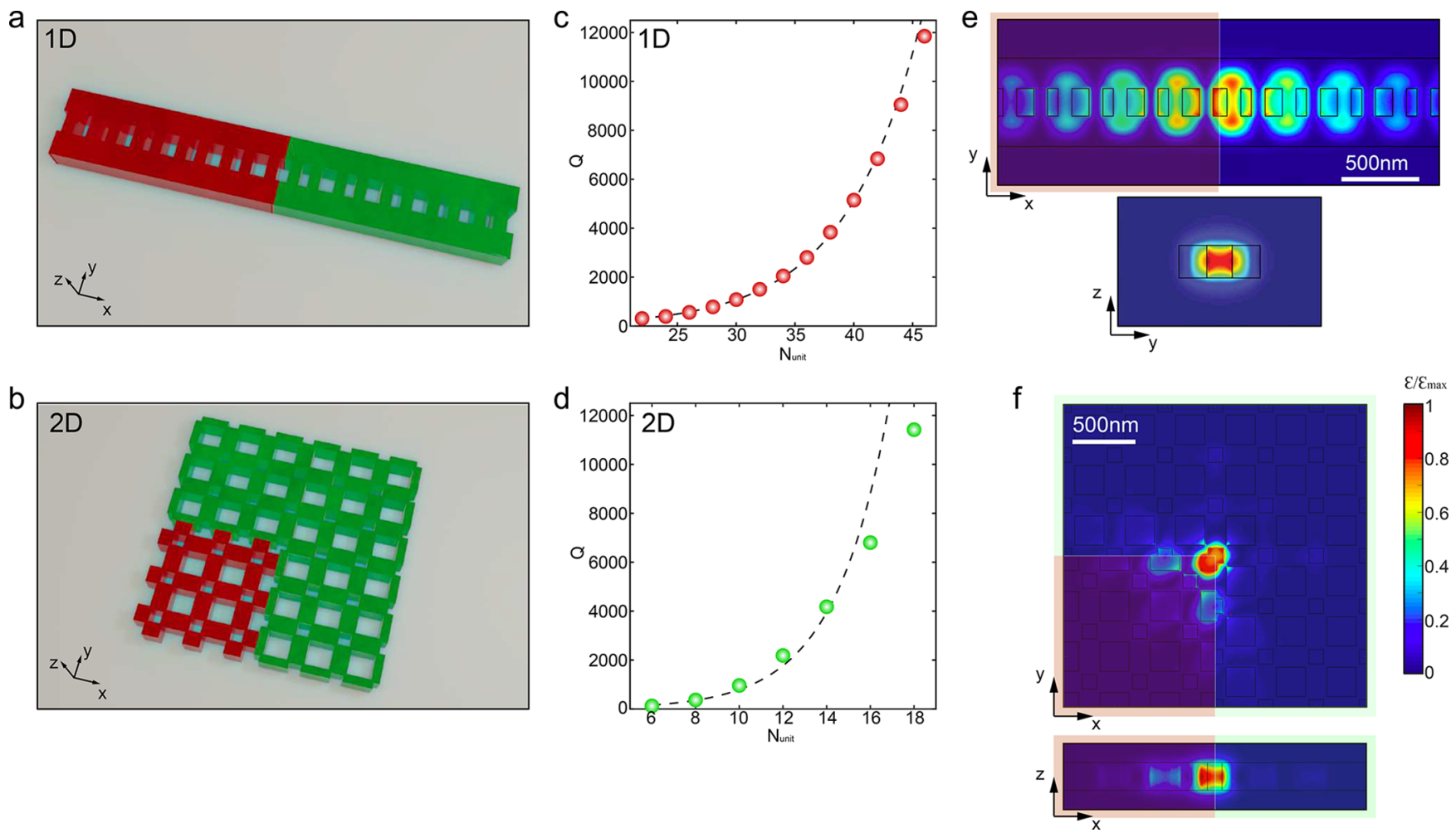

Figure 1. Hotpots generated in 1D/2D topological structures with high quality factor. (a, b) Schematic of (a) 1D topological structures and (b) 2D topological structures. Green and red represent the components with different values of topological invariants Topological edge/corner states exists between the two. (c, d) Relationship between the number of unit cells $\left(N_{\text {unit }}\right)$ and the quality factor (Q) for (c) 1D topological structures and (d) 2D topological structures. The dashed lines are exponential fits. (e) Demonstration of the hotspot formed in 1D structure. The cross-sections of spatial distribution of electromagnetic energy $\mathcal{E}$ in $x-y$ plane and $y-z$ plane are shown, respectively. The directions of $x, y$, and $z$ are demonstrated in panel a. (f) Demonstration of the hotspot formed in 2D structure. Cross-sections in $x-y$ plane and $y-z$ plane are selected. The directions of $x, y$, and $z$ are demonstrated in panel $b$.

broken to improve the near field enhancement for molecular barcoding, ${ }^{12}$ despite the quality factor degradation. ${ }^{37}$

More importantly, the resonance-based dielectric structures are vulnerable to fabrication imperfections (or geometric disorder), ${ }^{38-43}$ with a drastic performance degradation when realistic geometries deviates from the design. For example, only a few percent of disorder can eliminate the band gap of inverse opal photonic crystals. ${ }^{38,39}$ Good robustness only exists in limited cases including merging topological charges of BIC structures $^{44}$ or increasing the refractive indices of photonic crystals. $^{45}$

The exciting advances in topological photonics ${ }^{46-52}$ may shed light on a new platform for hotspots formed in the vicinity of dielectric structures. For a phase with bandgap, topological edge states are automatically formed at boundaries between bulks with different topological invariants, localizing photons where the Zak phase or Chern number jumps. The edge states not only show robustness to local geometric perturbation and defects but also are insensitive to moderate global disorder in the whole system including the bulk. Recently, the robustness of topological edges has been demonstrated both numerically ${ }^{53-56}$ and experimentally. ${ }^{57,58}$ In this article, we propose and numerically realize two types of hotspots with good robustness to geometric disorder, $1 \mathrm{D}$ edge states and $2 \mathrm{D}$ corner states in photonic topological structures, respectively. The field intensity of the hotspot increases simultaneously with the quality factor by enlarging the size of the systems. While $1 D$ edge states ${ }^{59-62}$ and $2 \mathrm{D}$ corner states ${ }^{63-66}$ have remained two isolated topics, we provide the first comparison of the two, to the best of our knowledge. This comprehensive robustness analysis will not only be a guide for experimental realization of topological states in optical regime where the fabrication imperfections are inevitable but also provide a novel platform for various hotspot-based applications mentioned earlier.

\section{RESULTS}

Recent progress of high order topological insulators ${ }^{67-71}$ provides three solutions to localize optical field in a spot, that is, a zero-dimensional (OD) geometry. Besides the edge state from a first-order one-dimensional (1D) topological structure, a OD spot can be achieved from second-order or even third-order topological phases with higher geometric dimensions. Considering the feasibility of fabrication, we focus on two types of topology-induced hotspots, edge states from 1D structures and corner states from 2D structures, instead of impractical corner states from third-order 3D topological phases.

We choose similar morphologies for $1 \mathrm{D}$ and $2 \mathrm{D}$ systems, a dielectric slab with designed rectangular air holes, as demonstrated in Figure 1a for a 1D structure and Figure 1b for a $2 \mathrm{D}$ structure. In both cases, topological boundary states are formed by connecting two types of periodic structures with different quantized Zak phases ${ }^{72}$ in either $1 \mathrm{D}^{73}$ or $2 \mathrm{D}^{74,75}$ structures. While the physical mechanism of the formation of the $1 \mathrm{D}^{59}$ or $2 \mathrm{D}^{63-65,76}$ topological states has been studied based on the Su-Schrieffer-Heeger model, respectively, we concentrate on comparing the hotspots produced by these two structures.

For the sake of a reasonable comparison, we fix the resonance of the topology-induced air cavity to the same spectral position 
by tuning the geometric parameters of the unit cells: $1540.8 \mathrm{~nm}$ in $1 \mathrm{D}$ versus $1542.2 \mathrm{~nm}$ in $2 \mathrm{D}$. The details of the parameters for both the $1 \mathrm{D}$ and 2D structures can be found in Supporting Information 1. An FDTD method is implemented to investigate the electromagnetic dynamics, with details shown in the Methods section and Supporting Information 2. The jump of topological invariants at the boundary enforces the localization of photons, forming an optical cavity filled with air, or equivalently a bright hotspot to interact with other materials inside or nearby. Figure $1 \mathrm{c}$ and $\mathrm{d}$ show the relationship between the quality factor $(Q)$ of the topological cavity and the number of unit cells along $x$-direction $\left(N_{\text {unit }}\right)$. For $2 \mathrm{D}$ structures, the numbers of unit cells in $x$ - and $y$-directions are equal. In both 1D and $2 \mathrm{D}$ structures, the number of unit cells with different Zak phases is identical, as shown in Figures $1 \mathrm{a}$ and $\mathrm{b}$ with red and green colors. As we extend the size of the structures, better confinement is achieved in both cases, verified by the increment of $Q$ accordingly. Owing to the additional confinement along the $y$ direction, 2D structures require fewer unit cells for the same level of $Q$. When the size of the structure is not too large, an exponential increase of $Q$ with $N_{\text {unit }}$ is observed, as shown by the fit shown in Figure 1c and d (dashed lines). The enhancement saturates when the size further increases $\left(N_{\text {unit }}>42\right.$ in $1 \mathrm{D}$ and $N_{\text {unit }}>14$ in 2D). We demonstrate this effect with larger values of $N_{\text {unit }}$ in Supporting Information 3.

For optical cavities under continuous-wave excitation, the maximum achievable energy increases with its $Q^{77,78}$ and the maximum light intensity inside is enhanced correspondingly. Thus, the topological cavity here provides a solution for hotspots with simultaneous increment in both quality factor and nearfield enhancement. By increasing the value of $N_{\text {unit }}$ the enhancement of $Q$ and near-field intensity can be readily achieved at the same time. Therefore, we treat the value of $Q$ as a figure of merit to describe the quality of the hotspots produced by the topological structures.

We select $N_{\text {unit }}$ of each structure so that the quality factors have similar and high enough values, that is, $Q_{1 D}\left(N_{\text {unit }}=46\right)=11$ 850 and $Q_{2 D}\left(N_{\text {unit }}=18\right)=11419$. Figure le and $f$ are crosssections of the spatial distribution of energy density along different planes, illustrating the hotspots produced in $1 \mathrm{D}$ and $2 \mathrm{D}$ structures separately. The energy density $\mathcal{E}$ includes both the contributions from electric field $(E)$ and magnetic field $(H)$, $\mathcal{E}=\frac{1}{2}\left(\varepsilon|E|^{2}+\mu|H|^{2}\right)$. Bright 3D hotspots in air are produced in both cases, efficiently localizing and enhancing electric fields in the center of the structures where a sharp change of topological invariants occurs. Also, due to breaking of symmetry, the energy density of the hotspots is not equally distributed. We attribute a better confinement of the $2 \mathrm{D}$ hotspots to the additional confinement in the $y$-direction.

Starting from the ideal (ordered) structures demonstrated in Figure 1, we scrutinize how the disorder impacts the quality of hotspots produced inside. We classify the disorder into two types, one in position and another in size, as demonstrated in Figure 2. Figure $2 \mathrm{a}$ is a schematic of a $2 \mathrm{D}$ structure without disorder. The disorder in size is introduced by varying the lengths in both $x$ - and $y$-direction of rectangular air holes, as shown in Figure $2 \mathrm{~b}$. The disorder in position represents the deviation of air holes away from their original (periodic) positions, as shown in Figure $2 \mathrm{c}$. Figure $2 \mathrm{~d}$ illustrates the case when disorder in position and size coexists. The degree of disorder is characterized by maximum fluctuations in size $\left(\delta_{\text {siz }}\right)$ and maximum deviations from original positions $\left(\delta_{\text {pos }}\right)$. Both

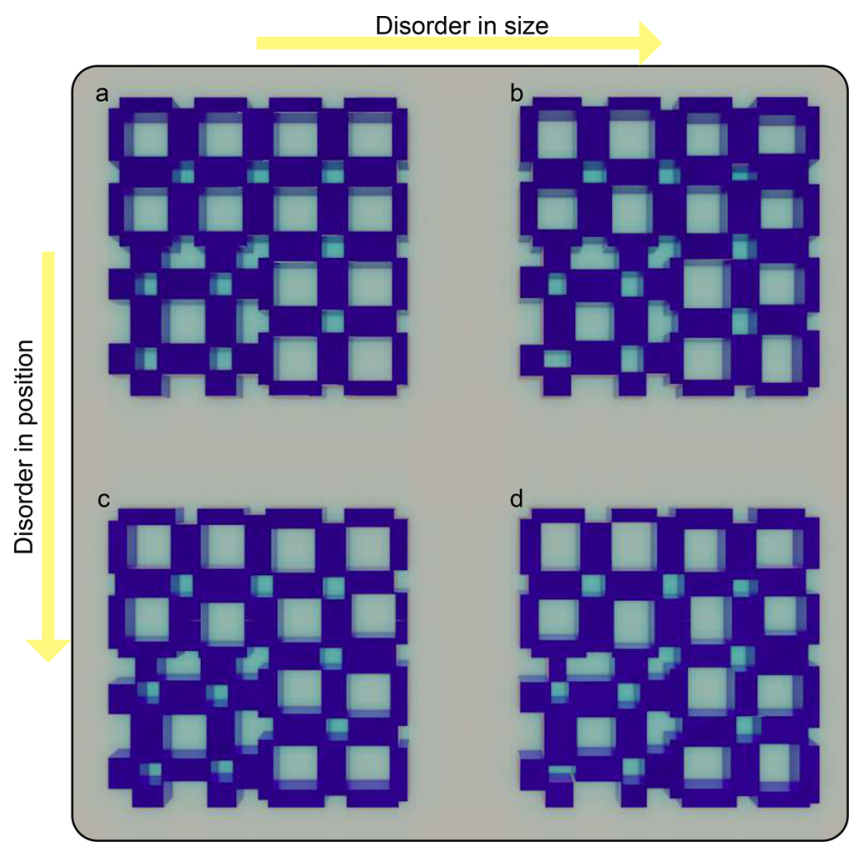

Figure 2. Demonstration of disorder in size $\delta_{\text {siz }}$ and in position $\delta_{\text {pos. }}$ (a) Ideal 2D structure without disorder. (b) 2D structure with fluctuation in the sizes of air holes; $\delta_{\text {siz }}=7 \%$ and $\delta_{\text {pos }}=0 \%$. (c) 2D structure with misalignment away from original positions; $\delta_{\text {siz }}=0 \%$ and $\delta_{\text {pos }}=7 \%$. (d) $2 \mathrm{D}$ structure with both disorder in shape and position; $\delta_{\text {siz }}=7 \%$ and $\delta_{\text {pos }}$ $=7 \%$.

values are normalized to the lattice constant of the unit cell $a_{0}$. Figure $2 \mathrm{~b}$ and $\mathrm{c}$ represent the values of $\delta_{\text {siz }}=7 \%$ and $\delta_{\text {pos }}=7 \%$, respectively. More details can be found in Supporting Information 2.

First, we investigate the influence of disorder in size on the robustness of the hotspots produced by both $1 \mathrm{D}$ and $2 \mathrm{D}$ structures, with results summarized in Figure 3. In Figure 3a and $\mathrm{b}$, the relationships between $Q$ and $\delta_{\text {siz }}$ are demonstrated, respectively. The value of the quality factor is normalized to its value without disorder $\left(Q_{0}\right)$. Furthermore, it is averaged from five batches of simulations with different sets of random variables. The shaded areas in Figure $3 a$ and $b$ represent the standard deviation. The dashed lines in Figure $3 a$ and $b$ are identical, acting as a guide for comparison. Interestingly, the size disorder has almost the same impact on the degradation of the value of $Q$ in $1 D$ and $2 D$ structures. In the investigated geometries, the quality factor can preserve $40 \%$ of its original value under $5 \%$ of disorder. Figure $3 \mathrm{c}$ and $\mathrm{d}$ show typical sets of energy density spectra of the topological modes under different $\delta_{\text {siz }}$. The spectrum is obtained from the Fourier transform of the time evolution of energy density from points inside the air cavity. The maximum value is normalized to one. Disorder not only reduces the quality factor of the modes but also perturbs the resonant peaks (represented by gray dots in the figures) in both $1 \mathrm{D}$ and $2 \mathrm{D}$ structures. Both blue shifts and red shifts are observed depending on different random sets in both cases. Figure $3 \mathrm{e}$ and $\mathrm{f}$ illustrate the influence of the disorder $\delta_{\text {siz }}$ on the spatial distribution of the energy density at the resonance. To compare modes with different values of $Q$, we normalize the energy density $\mathcal{E}$ to its maximum value $\mathcal{E}_{\max }$. Although the photons are less confined as the amount of disorder increases, hotspots exist in the center for both $1 \mathrm{D}$ and $2 \mathrm{D}$ structures. The field enhancement of both $1 \mathrm{D}$ and 2D structures can be found in Supporting Information 4. 

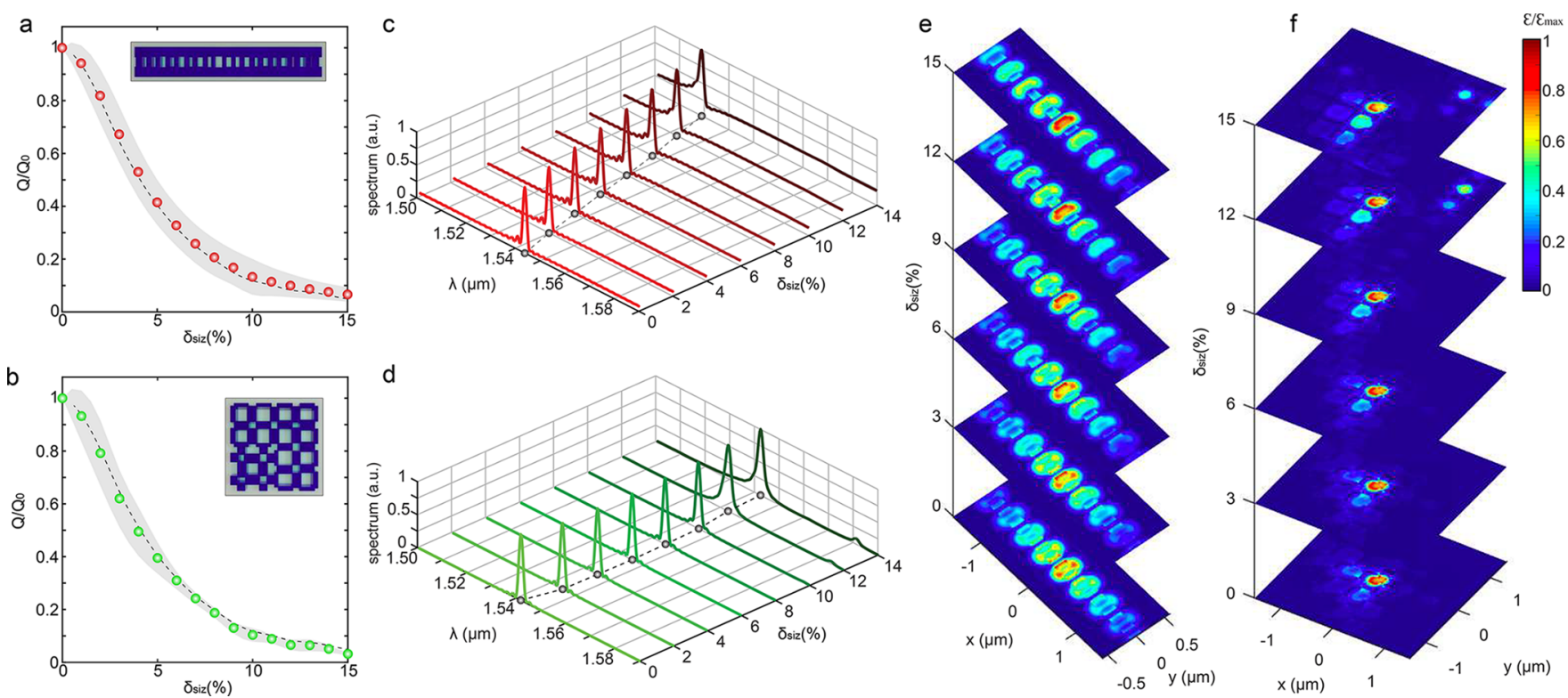

Figure 3. Robustness investigation of hotspots with disorder in size $\delta_{\text {siz }}$ (a, b) Relationship between normalized quality factor $Q / Q_{0}$ and $\delta_{\text {siz }}$ in $(a) 1 D$ structures and (b) 2D structures. Value of $Q$ is averaged from five different sets of random variables. The shaded areas represent the standard deviation. The insets show schematic of structures with disorder in size. $(c, d)$ Energy spectra of the topological states with different values of $\delta_{\text {siz }}$ from $(c) 1 D$ structures and (d) 2D structures. The gray dots show the spectral position of the resonance. (e, f) Cross-sections of spatial distribution of the energy density with different values of $\delta_{\text {siz }}$ from (e) $1 \mathrm{D}$ structures and (f) $2 \mathrm{D}$ structures.
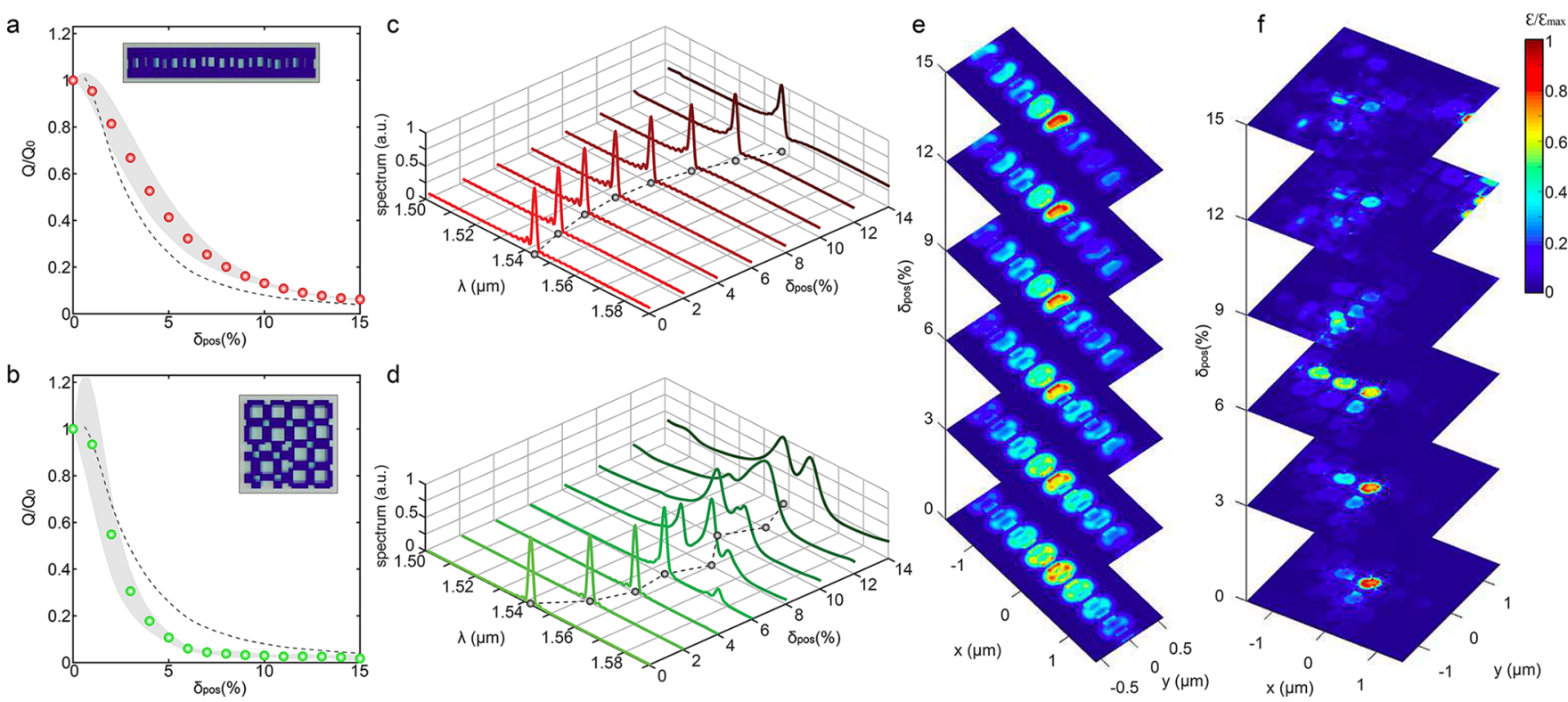

Figure 4. Robustness investigation of hotspots with disorder in position $\delta_{\text {pos }}$ (a, b) Relationship between normalized quality factor $Q / Q_{0}$ and $\delta_{\text {pos }}$ in (a) 1D structures and (b) 2D structures. The value of $Q$ is averaged from five different sets of random variables. The shaded areas represent the standard deviation. The insets show schematic of structures with disorder in position. (c, d) Energy spectra of the topological stages with different values of $\delta_{\text {pos }}$ from (c) 1D structures and (d) 2D structures. The gray dots show the spectral position of the resonance. (e) Cross-sections of spatial distribution of the energy density with different values of $\delta_{\text {pos }}$ from (e) $1 \mathrm{D}$ structures and (f) $2 \mathrm{D}$ structures.

Figure 4 provides a comparison of the robustness of hotspots with disorder in position $\delta_{\text {pos. }}$. Figure $4 \mathrm{a}$ and $\mathrm{b}$ show the relationship between $Q$ and $\delta_{\text {poss }}$. The robustness values in $1 \mathrm{D}$ and $2 \mathrm{D}$ systems are quite different, in contrast to the similar behavior shown in Figure 3a and b. While the 2D structures have a better localization of electromagnetic energy, they are more vulnerable to fluctuations of the position of air holes. The $1 \mathrm{D}$ structure has better robustness, with the averaged value of quality factor always larger than the $2 \mathrm{D}$ counterpart. In both $1 \mathrm{D}$ and $2 \mathrm{D}$ structures with a small amount of disorder $\left(\delta_{\text {pos }}=1 \%\right.$ or $\delta_{\text {siz }}=1 \%$ ), the quality factor is boosted in some configurations, inducing large values of the standard deviation (shaded area) and values of $Q / Q_{0}$ beyond one (as shown in Figures 3 and 4). This phenomenon of disorder-induced confinement enhancement was observed previously. ${ }^{45,79}$ We attribute it to the fact that the disorder-free structures may not be optimized solutions ${ }^{80}$ for the maximized quality factor. Meanwhile, the Anderson localization may not be the driving force here, considering the small amount of disorder. ${ }^{81}$ However, the averaged value from five different random sets is smaller than $Q_{0}$, 

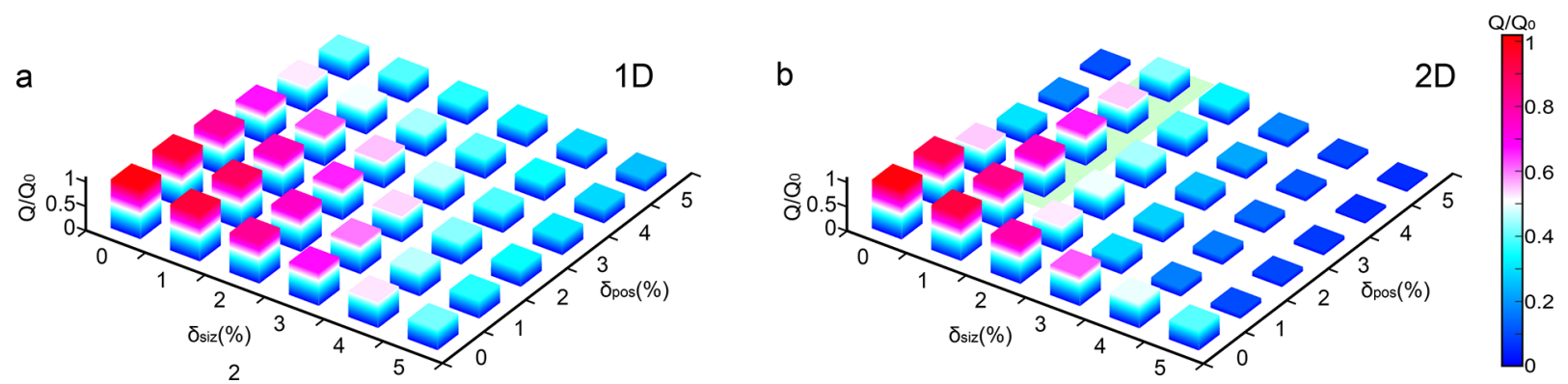

Figure 5. Robustness investigation of hotspots with disorder in both size $\delta_{\text {siz }}$ and position $\delta_{\text {pos. }}$. The height and color of the pillars represent the normalized quality factor under different levels of disorder for (a) 1D structures and (b) 2D structures. A better robustness is achieved in 1D structures.
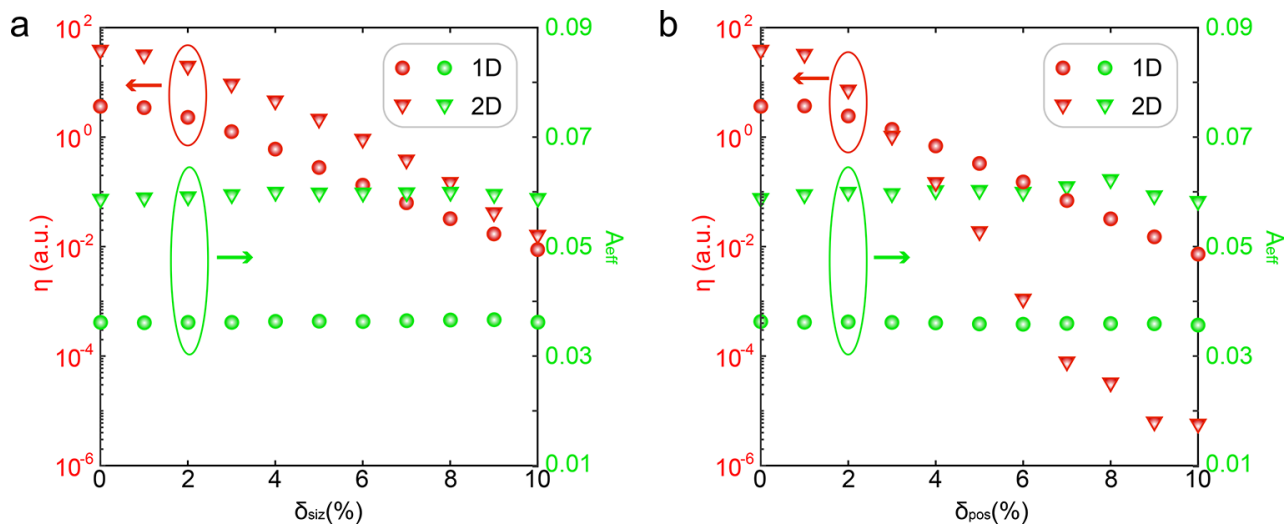

Figure 6. Comparison of the figure of merit $\eta$ and effective area $A_{\text {eff }}$ for topological hotspots between $1 \mathrm{D}$ and $2 \mathrm{D}$ structures with disorder. (a) Comparison with disorder in size $\delta_{\text {siz. }}$. (b) Comparison with disorder in position $\delta_{\text {pos. }}$. All results are averaged from five different sets of random variables.

with more details shown in Supporting Information 5 for 2D structures with disorder in position. Similarly, we compare energy density spectra of the topological modes in Figure 4c (1D) and d (2D). Despite the degradation of quality factor (or the broadening of the full width half-maximum) with increase of disorder, a single mode dominates in 1D structures. Correspondingly, hotspots at the center of the structure preserve under the disorder, as illustrated in Figure 4e. In contrast, the disorder in position strongly perturbs the topological mode in 2D structures. The misalignment of air holes breaks the symmetry, and additional modes are observed when $\delta_{\text {pos }}>6 \%$. The hotspot in the center disappears when misalignment $\delta_{\text {pos }}$ increases, as demonstrated in Figure 4f. The field enhancement of both 1D and 2D structures can be found in Supporting Information 4.

On the basis of numerical simulations, we observe better robustness in $1 \mathrm{D}$ topological structures than the $2 \mathrm{D}$ counterpart and also a stronger impact from disorder in position. We may explain the effect qualitatively from the tight-binding model. There are twice nearest neighbors in $2 \mathrm{D}$ than $1 \mathrm{D}$ ( 4 vs 2 ). Meanwhile, the distance to the second nearest neighbors is also smaller in 2D structures. Therefore, $2 \mathrm{D}$ structures may be more vulnerable to fluctuations. The fluctuation in size may have a smaller impact on the coupling strength than directly randomized shifts in position. Consequently, the structures are more robust under the disorder in size.

Next, we investigate structures with embedded disorder in both size and position, close to realistic devices with fabrication imperfection. Figure 5 shows how the hybrid disorder impacts hotspots. Again, the values are averaged from five different random sets and normalized to the value without disorder. Figure $5 \mathrm{a}$ demonstrates the robustness of $1 \mathrm{D}$ structures. The normalized quality factor demonstrates a good symmetry along the diagonal $\left(\delta_{\text {siz }}=\delta_{\text {pos }}\right)$, implying that the shape fluctuation and misalignment of air holes induce a similar impact on the hotspots. If fluctuations in both the shape and position of air holes are kept within 3\%, the 1D configuration can maintain 50\% of the quality factor. The robustness of the $2 \mathrm{D}$ structures is quite different from its $1 \mathrm{D}$ counterpart, with results presented in Figure $5 \mathrm{~b}$. The degradation of $Q$ is more rapid as $\delta_{\text {pos }}$ increases, indicating that the system is more fragile to the misalignment of air holes. We attribute this feature to the fact that the fluctuation in position may intensively exacerbate the periodicity, other than the fluctuation in size where the centers of air holes do not shift. This feature makes the 2D structure less robust than its $1 \mathrm{D}$ counterpart when disorder in size and position coexists. We also observe an area (the light green region in Figure $5 b$ ) where the value of $Q$ increase with the introduction of $\delta_{\text {siz }}$ from $0 \%$ to $1 \%$. However, the area is very limited when misalignment and a minute fluctuation of the shape $\left(\delta_{\text {siz }}=1 \%\right)$ coexists. The value of $Q$ decreases rapidly with the further increment of the disorder.

Besides the quality factor, other properties such as the energy density (or light intensity) and the size of hotspots are also important parameters for practical applications. We define another figure of merit $\eta$ to characterize the hotspot, which is the multiplication of the quality factor and averaged energy density in the air cavity; $\eta=Q \overline{\mathcal{E}}$. Meanwhile, we define an effective area of the hotspot, which is the area in the cavity where its energy density is larger than the average value. To make a reasonable comparison, we normalize the effective area by the area of the unit cell $\left(A_{0}\right) ; A_{\text {eff }}=\iint_{\mathcal{E}>\overline{\mathcal{E}}} \mathrm{d} x \mathrm{~d} y / A_{0}$. We summarize the results in Figure 6, demonstrating the impact of disorder in size $\left(\delta_{\text {siz }}\right)$ and disorder in position $\left(\delta_{\text {pos }}\right)$ in a and b, respectively. The 
effective area $A_{\text {eff }}$ shows similar values under moderate level of disorder in both cases, owing to the protection from topology. The additional confinement along the $y$ axis endows the 2D structures a larger $A_{\text {eff }}$ than their 1D counterparts (6.0\% vs $3.6 \%$ of the unit cell area). Although the $1 \mathrm{D}$ and $2 \mathrm{D}$ structures share similar values of $Q$ under the disorder in size, the additional confinement in 2D induces larger energy density $\overline{\mathcal{E}}$. As a result, the value of $\eta$ is larger in 2D structures when $\delta_{\text {siz }}$ is small, as shown in Figure 6a. However, the 1D structures bare better robustness; the difference of $\eta$ between $1 \mathrm{D}$ and 2D structures shirks as $\delta_{\text {siz }}$ increases. The robustness of $1 \mathrm{D}$ structures to the disorder in position is more prominent, as shown in Figure $6 \mathrm{~b}$. The value of $\eta$ in 2D structures decreases dramatically with the increment of $\delta_{\text {pos }}$, making 1D structures a better choice when the moderate misalignment of the geometry exists.

\section{DISCUSSION}

While the intense light localization of topological states either in $1 \mathrm{D}$ or $2 \mathrm{D}$ structures has been investigated, previous studies focused on the light-matter interaction in the dielectric part for applications including lasing and nonlinear generation. ${ }^{59,60,62,64-66}$ Here, we propose to leverage the topological edge/corner states to produce hotspots in air, which may inspire a repertoire of novel applications ranging from sensing to photocatalysis. The light confinement is induced by a sharp change of the topological invariant at boundaries, enforcing strong confinement in both air and dielectric. By simply increasing the number of unit cells, the light intensity and quality factor (or equivalent sensitivity) of hotspots can be boosted concomitantly, mitigating the mismatch between quality factor and near-field enhancement of dielectric resonators.

To provide a guide for potential applications, we scrutinize and compare the robustness of topological states in 1D and 2D structures. While the additional dimension of $2 \mathrm{D}$ structures offers better confinement, more uniform intensity, and larger effective area of the hotspots (as shown in Figures 1e,f), the hotspots formed in 1D structures are more robust to fabrication errors including the fluctuations in the shape and misalignment of desired patterns. Also, to achieve the same quality factor, 1D structures consume less footprint than the $2 \mathrm{D}$ counterpart (46 units cell for $1 \mathrm{D}$ vs 324 unit cells for $2 \mathrm{D}$ for a $Q$ of $1.15 \times 10^{4}$ ). Besides some applications requiring a good uniformity of hotspots, the $1 \mathrm{D}$ topological structure is recommended for the exploration of emerging applications by leveraging the bright, robust, and high quality hotspots. In the simulations, we model the dielectric with a real refractive index, and material loss (absorption) is neglected. The introduction of loss can induce the degradation of the quality factor. However, the topology induced robustness still exists, showing the applicability for practical devices. More details can be found in Supporting Information 6.

\section{METHODS}

All simulations are based on the FDTD method by a commercialized software (LUMERICAL, FDTD Solution). The refractive index of the dielectric is fixed to 3.3 , corresponding to the value of InGaAsP. The quality factor is simulated with the analysis group " $Q$ analysis" provided by LUMERICAL. More details can be found in Supporting Information 2.

\section{ASSOCIATED CONTENT}

\section{(s) Supporting Information}

The Supporting Information is available free of charge at https://pubs.acs.org/doi/10.1021/acsphotonics.1c01445.

Geometry of topological structures; details of FDTD simulations; saturation of quality factor as extension of structural size; field-enhancement under disorder; details of large fluctuation of quality factor with small disorder in position; hotspots generated from materials with loss (PDF)

\section{AUTHOR INFORMATION}

\section{Corresponding Authors}

Changxu Liu - Chair in Hybrid Nanosystems, Nanoinstitute Munich, Faculty of Physics, Ludwig Maximilians University of Munich, 80539 Munich, Germany; Department of Mathematics, Physics and Electrical Engineering, Northumbria University, Newcastle Upon Tyne NE1 8ST, United Kingdom; (1) orcid.org/0000-0003-1196-7447; Email: changxu.liu@ physik.uni-muenchen.de

Stefan A. Maier - Chair in Hybrid Nanosystems, Nanoinstitute Munich, Faculty of Physics, Ludwig Maximilians University of Munich, 80539 Munich, Germany; Department of Physics, Imperial College London, London SW7 2AZ, United Kingdom; 이이.org/0000-0001-9704-7902; Email: stefan.maier@physik.uni-muenchen.de

Complete contact information is available at: https://pubs.acs.org/10.1021/acsphotonics.1c01445

\section{Funding}

C.L. acknowledges the funding support from Humboldt Research Fellowship from the Alexander von Humboldt Foundation. S.A.M. acknowledges the funding support from the Deutsche Forschungsgemeinschaft (DFG, German Research Foundation) under Germany's Excellence Strategy, EXC 2089/1-390776260, the Solar Energies go Hybrid (SolTech) program and Lee-Lucas Chair in Physics.

\section{Notes}

The authors declare no competing financial interest.

\section{REFERENCES}

(1) Aouani, H.; Rahmani, M.; Navarro-Cía, M.; Maier, S. A. Thirdharmonic-upconversion enhancement from a single semiconductor nanoparticle coupled to a plasmonic antenna. Nat. Nanotechnol. 2014, 9, 290.

(2) Li, G.; Zhang, S.; Zentgraf, T. Nonlinear photonic metasurfaces. Nat. Rev. Mater. 2017, 2, 17010.

(3) Atwater, H. A.; Polman, A. Plasmonics for improved photovoltaic devices. Nat. Mater. 2010, 9, 205-213.

(4) Ma, C.; Liu, C.; Huang, J.; Ma, Y.; Liu, Z.; Li, L.-J.; Anthopoulos, T. D.; Han, Y.; Fratalocchi, A.; Wu, T. Plasmonic-enhanced light harvesting and perovskite solar cell performance using Au biometric dimers with broadband structural darkness. Sol. RRL 2019, 3, 1900138.

(5) Caldarola, M.; Albella, P.; Cortés, E.; Rahmani, M.; Roschuk, T.; Grinblat, G.; Oulton, R. F.; Bragas, A. V.; Maier, S. A. Non-plasmonic nanoantennas for surface enhanced spectroscopies with ultra-low heat conversion. Nat. Commun. 2015, 6, 7915.

(6) Alessandri, I.; Lombardi, J. R. Enhanced Raman scattering with dielectrics. Chem. Rev. 2016, 116, 14921-14981.

(7) Mao, P.; Liu, C.; Favraud, G.; Chen, Q.; Han, M.; Fratalocchi, A.; Zhang, S. Broadband single molecule SERS detection designed by warped optical spaces. Nat. Commun. 2018, 9, 5428. 
(8) Bell, S. E.; Charron, G.; Cortés, E.; Kneipp, J.; de la Chapelle, M. L.; Langer, J.; Procházka, M.; Tran, V.; Schlücker, S. Towards reliable and quantitative surface-enhanced Raman scattering (SERS): From key parameters to good analytical practice. Angew. Chem., Int. Ed. Engl. 2020, 59, 5454-5462.

(9) Mao, P.; Liu, C.; Chen, Q.; Han, M.; Maier, S. A.; Zhang, S. Broadband SERS detection with disordered plasmonic hybrid aggregates. Nanoscale 2020, 12, 93-102.

(10) Fenzl, C.; Hirsch, T.; Wolfbeis, O. S. Photonic crystals for chemical sensing and biosensing. Angew. Chem., Int. Ed. 2014, 53, $3318-3335$.

(11) Pitruzzello, G.; Krauss, T. F. Photonic crystal resonances for sensing and imaging. J. Opt. 2018, 20, 073004.

(12) Tittl, A.; Leitis, A.; Liu, M.; Yesilkoy, F.; Choi, D.-Y.; Neshev, D. N.; Kivshar, Y. S.; Altug, H. Imaging-based molecular barcoding with pixelated dielectric metasurfaces. Science 2018, 360, 1105-1109.

(13) Yesilkoy, F.; Arvelo, E. R.; Jahani, Y.; Liu, M.; Tittl, A.; Cevher, V.; Kivshar, Y.; Altug, H. Ultrasensitive hyperspectral imaging and biodetection enabled by dielectric metasurfaces. Nat. Photonics 2019, 13, 390-396.

(14) Jahani, Y.; Arvelo, E. R.; Yesilkoy, F.; Koshelev, K.; Cianciaruso, C.; De Palma, M.; Kivshar, Y.; Altug, H. Imaging-based spectrometerless optofluidic biosensors based on dielectric metasurfaces for detecting extracellular vesicles. Nat. Commun. 2021, 12, 3246.

(15) Foreman, M. R.; Swaim, J. D.; Vollmer, F. Whispering gallery mode sensors. Adv. Opt. Photonics 2015, 7, 168-240.

(16) Bardhan, R.; Grady, N. K.; Cole, J. R.; Joshi, A.; Halas, N. J. Fluorescence enhancement by $\mathrm{Au}$ nanostructures: nanoshells and nanorods. ACS Nano 2009, 3, 744-752.

(17) Acuna, G.; Möller, F.; Holzmeister, P.; Beater, S.; Lalkens, B.; Tinnefeld, P. Fluorescence enhancement at docking sites of DNAdirected self-assembled nanoantennas. Science 2012, 338, 506-510.

(18) Wang, Z.; Dong, Z.; Gu, Y.; Chang, Y.-H.; Zhang, L.; Li, L.-J.; Zhao, W.; Eda, G.; Zhang, W.; Grinblat, G.; Maier, S. A.; Yang, J. K. W.; Cheng-Wei, Q.; S, W. A. T. Giant photoluminescence enhancement in tungsten-diselenide-gold plasmonic hybrid structures. Nat. Commun. 2016, 7, 11283 .

(19) Regmi, R.; Berthelot, J.; Winkler, P. M.; Mivelle, M.; Proust, J.; Bedu, F.; Ozerov, I.; Begou, T.; Lumeau, J.; Rigneault, H.; GarcíaParajó, M. F.; Bidault, S.; Wenger, J.; Bonod, N. All-dielectric silicon nanogap antennas to enhance the fluorescence of single molecules. Nano Lett. 2016, 16, 5143-5151.

(20) Vaskin, A.; et al. Manipulation of magnetic dipole emission from Eu3+ with Mie-resonant dielectric metasurfaces. Nano Lett. 2019, 19, $1015-1022$.

(21) Cortés, E.; Xie, W.; Cambiasso, J.; Jermyn, A. S.; Sundararaman, R.; Narang, P.; Schlücker, S.; Maier, S. A. Plasmonic hot electron transport drives nano-localized chemistry. Nat. Commun. 2017, 8, 14880 .

(22) Wang, X.; et al. Self-Constructed Multiple Plasmonic Hotspots on an Individual Fractal to Amplify Broadband Hot Electron Generation. ACS Nano 2021, 15, 10553-10564.

(23) Cortés, E.; Besteiro, L. V.; Alabastri, A.; Baldi, A.; Tagliabue, G.; Demetriadou, A.; Narang, P. Challenges in Plasmonic Catalysis. ACS Nano 2020, 14, 16202-16219.

(24) Camargo, P. H.; Cortés, E. Plasmonic Catalysis: From Fundamentals to Applications; Wiley-VCH: Weinheim, 2021.

(25) Shoji, T.; Tsuboi, Y. Plasmonic optical tweezers toward molecular manipulation: tailoring plasmonic nanostructure, light source, and resonant trapping. J. Phys. Chem. Lett. 2014, 5, 2957-2967.

(26) Ndukaife, J. C.; Kildishev, A. V.; Nnanna, A. G. A.; Shalaev, V. M.; Wereley, S. T.; Boltasseva, A. Long-range and rapid transport of individual nano-objects by a hybrid electrothermoplasmonic nanotweezer. Nat. Nanotechnol. 2016, 11, 53.

(27) Maier, S. A. Plasmonics: Fundamentals and Applications; Springer Science \& Business Media, 2007.

(28) Khurgin, J. B.; Boltasseva, A. Reflecting upon the losses in plasmonics and metamaterials. MRS Bull. 2012, 37, 768-779.
(29) Aubin-Tam, M.-E.; Hwang, W.; Hamad-Schifferli, K. Sitedirected nanoparticle labeling of cytochrome c. Proc. Natl. Acad. Sci. U. S. A. 2009, 106, 4095-4100.

(30) Mahmoudi, M.; Lohse, S. E.; Murphy, C. J.; Fathizadeh, A.; Montazeri, A.; Suslick, K. S. Variation of protein corona composition of gold nanoparticles following plasmonic heating. Nano Lett. 2014, 14, 6-12.

(31) Blázquez-Castro, A. Optical tweezers: phototoxicity and thermal stress in cells and biomolecules. Micromachines 2019, 10, 507.

(32) Kuznetsov, A. I.; Miroshnichenko, A. E.; Brongersma, M. L.; Kivshar, Y. S.; Luk'yanchuk, B. Optically resonant dielectric nanostructures. Science 2016, 354, 846.

(33) Hsu, C. W.; Zhen, B.; Stone, A. D.; Joannopoulos, J. D.; Soljačí́, M. Bound states in the continuum. Nat. Rev. Mater. 2016, 1, 1-13.

(34) Koshelev, K.; Bogdanov, A.; Kivshar, Y. Meta-optics and bound states in the continuum. Sci. Bull. 2019, 64, 836-842.

(35) Conteduca, D.; Barth, I.; Pitruzzello, G.; Reardon, C. P.; Martins, E. R.; Krauss, T. F. Dielectric nanohole array metasurface for highresolution near-field sensing and imaging. Nat. Commun. 2021, 12, 3293.

(36) Liang, Y.; Koshelev, K.; Zhang, F.; Lin, H.; Lin, S.; Wu, J.; Jia, B.; Kivshar, Y. Bound states in the continuum in anisotropic plasmonic metasurfaces. Nano Lett. 2020, 20, 6351-6356.

(37) Koshelev, K.; Lepeshov, S.; Liu, M.; Bogdanov, A.; Kivshar, Y. Asymmetric Metasurfaces with High- $Q$ Resonances Governed by Bound States in the Continuum. Phys. Rev. Lett. 2018, 121, 193903.

(38) Li, Z.-Y.; Zhang, Z.-Q. Photonic Bandgaps in Disordered InverseOpal Photonic Crystals. Adv. Mater. 2001, 13, 433-436.

(39) Li, Z.-Y.; Zhang, Z.-Q. Fragility of photonic band gaps in inverseopal photonic crystals. Phys. Rev. B: Condens. Matter Mater. Phys. 2000, $62,1516$.

(40) Kaliteevski, M.; Beggs, D.; Brand, S.; Abram, R.; Nikolaev, V. Stability of the photonic band gap in the presence of disorder. Phys. Rev. B: Condens. Matter Mater. Phys. 2006, 73, 033106.

(41) Lavrinenko, A. V.; Wohlleben, W.; Leyrer, R. J. Influence of imperfections on the photonic insulating and guiding properties of finite Si-inverted opal crystals. Opt. Express 2009, 17, 747-760.

(42) Ishizaki, K.; Okano, M.; Noda, S. Numerical investigation of emission in finite-sized, three-dimensional photonic crystals with structural fluctuations. J. Opt. Soc. Am. B 2009, 26, 1157-1161.

(43) Minkov, M.; Dharanipathy, U. P.; Houdré, R.; Savona, V. Statistics of the disorder-induced losses of high-Q photonic crystal cavities. Opt. Express 2013, 21, 28233-28245.

(44) Jin, J.; Yin, X.; Ni, L.; Soljačić, M.; Zhen, B.; Peng, C. Topologically enabled ultrahigh-Q guided resonances robust to out-ofplane scattering. Nature 2019, 574, 501-504.

(45) Liu, C.; Rybin, M. V.; Mao, P.; Zhang, S.; Kivshar, Y. DisorderImmune Photonics Based on Mie-Resonant Dielectric Metamaterials. Phys. Rev. Lett. 2019, 123, 163901.

(46) Lu, L.; Joannopoulos, J. D.; Soljačić, M. Topological photonics. Nat. Photonics 2014, 8, 821-829.

(47) Khanikaev, A. B.; Shvets, G. Two-dimensional topological photonics. Nat. Photonics 2017, 11, 763-773.

(48) Ozawa, T.; Price, H. M.; Amo, A.; Goldman, N.; Hafezi, M.; Lu, L.; Rechtsman, M. C.; Schuster, D.; Simon, J.; Zilberberg, O.; Iacopo, C. Topological photonics. Rev. Mod. Phys. 2019, 91, 015006.

(49) Smirnova, D.; Leykam, D.; Chong, Y.; Kivshar, Y. Nonlinear topological photonics. Appl. Phys. Rev. 2020, 7, 021306.

(50) Segev, M.; Bandres, M. A. Topological photonics: Where do we go from here? Nanophotonics 2020, 10, 425.

(51) Kim, M.; Jacob, Z.; Rho, J. Recent advances in 2D, 3D and higher-order topological photonics. Light: Sci. Appl. 2020, 9, 1-30.

(52) Ota, Y.; Takata, K.; Ozawa, T.; Amo, A.; Jia, Z.; Kante, B.; Notomi, M.; Arakawa, Y.; Iwamoto, S. Active topological photonics. Nanophotonics 2020, 9, 547-567.

(53) Liu, C.; Gao, W.; Yang, B.; Zhang, S. Disorder-induced topological state transition in photonic metamaterials. Phys. Rev. Lett. 2017, 119, 183901. 
(54) Mansha, S.; Chong, Y. D. Robust edge states in amorphous gyromagnetic photonic lattices. Phys. Rev. B: Condens. Matter Mater. Phys. 2017, 96, 121405.

(55) Jin, Y.; Torrent, D.; Djafari-Rouhani, B. Robustness of conventional and topologically protected edge states in phononic crystal plates. Phys. Rev. B: Condens. Matter Mater. Phys. 2018, 98, 054307.

(56) Proctor, M.; Huidobro, P. A.; Bradlyn, B.; de Paz, M. B.; Vergniory, M. G.; Bercioux, D.; García-Etxarri, A. Robustness of topological corner modes in photonic crystals. Phys. Rev. Res. 2020, 2, 042038.

(57) Jiang, J.; Guo, Z.; Ding, Y.; Sun, Y.; Li, Y.; Jiang, H.; Chen, H. Experimental demonstration of the robust edge states in a split-ringresonator chain. Opt. Express 2018, 26, 12891-12902.

(58) Zhou, P.; Liu, G.-G.; Ren, X.; Yang, Y.; Xue, H.; Bi, L.; Deng, L.; Chong, Y.; Zhang, B. Photonic amorphous topological insulator. Light: Sci. Appl. 2020, 9, 1-8.

(59) Ota, Y.; Katsumi, R.; Watanabe, K.; Iwamoto, S.; Arakawa, Y. Topological photonic crystal nanocavity laser. Commun. Phys. 2018, 1, 86.

(60) Zhao, H.; Miao, P.; Teimourpour, M. H.; Malzard, S.; ElGanainy, R.; Schomerus, H.; Feng, L. Topological hybrid silicon microlasers. Nat. Commun. 2018, 9, 981.

(61) Parto, M.; Wittek, S.; Hodaei, H.; Harari, G.; Bandres, M. A.; Ren, J.; Rechtsman, M. C.; Segev, M.; Christodoulides, D. N.; Khajavikhan, M. Edge-mode lasing in 1D topological active arrays. Phys. Rev. Lett. 2018, 120, 113901.

(62) Kruk, S.; Poddubny, A.; Smirnova, D.; Wang, L.; Slobozhanyuk, A.; Shorokhov, A.; Kravchenko, I.; Luther-Davies, B.; Kivshar, Y. Nonlinear light generation in topological nanostructures. Nat. Nanotechnol. 2019, 14, 126-130.

(63) Ota, Y.; Liu, F.; Katsumi, R.; Watanabe, K.; Wakabayashi, K.; Arakawa, Y.; Iwamoto, S. Photonic crystal nanocavity based on a topological corner state. Optica 2019, 6, 786-789.

(64) Kim, H.-R.; Hwang, M.-S.; Smirnova, D.; Jeong, K.-Y.; Kivshar, Y.; Park, H.-G. Multipolar lasing modes from topological corner states. Nat. Commun. 2020, 11, 5758.

(65) Zhang, W.; Xie, X.; Hao, H.; Dang, J.; Xiao, S.; Shi, S.; Ni, H.; Niu, Z.; Wang, C.; Jin, K.; Zhang, X.; Xu, X. Low-threshold topological nanolasers based on the second-order corner state. Light: Sci. Appl. 2020, 9, 109.

(66) Kruk, S. S.; Gao, W.; Choi, D.-Y.; Zentgraf, T.; Zhang, S.; Kivshar, Y. Nonlinear Imaging of Nanoscale Topological Corner States. Nano Lett. 2021, 21, 4592-4597.

(67) Peterson, C. W.; Benalcazar, W. A.; Hughes, T. L.; Bahl, G. A quantized microwave quadrupole insulator with topologically protected corner states. Nature 2018, 555, 346-350.

(68) Noh, J.; Benalcazar, W. A.; Huang, S.; Collins, M. J.; Chen, K. P.; Hughes, T. L.; Rechtsman, M. C. Topological protection of photonic mid-gap defect modes. Nat. Photonics 2018, 12, 408-415.

(69) Mittal, S.; Orre, V. V.; Zhu, G.; Gorlach, M. A.; Poddubny, A.; Hafezi, M. Photonic quadrupole topological phases. Nat. Photonics 2019, 13, 692-696.

(70) El Hassan, A.; Kunst, F. K.; Moritz, A.; Andler, G.; Bergholtz, E. J.; Bourennane, M. Corner states of light in photonic waveguides. Nat. Photonics 2019, 13, 697-700.

(71) Xie, B.; Wang, H.-X.; Zhang, X.; Zhan, P.; Jiang, J.-H.; Lu, M.; Chen, Y. Higher-order band topology. Nat. Rev. Phys. 2021, 3, 520532.

(72) Zak, J. Berry?s phase for energy bands in solids. Phys. Rev. Lett. 1989, 62, 2747.

(73) Xiao, M.; Zhang, Z.; Chan, C. T. Surface impedance and bulk band geometric phases in one-dimensional systems. Phys. Rev. X 2014, 4, 021017.

(74) Liu, F.; Wakabayashi, K. Novel topological phase with a zero berry curvature. Phys. Rev. Lett. 2017, 118, 076803.

(75) Xie, B.-Y.; Wang, H.-F.; Wang, H.-X.; Zhu, X.-Y.; Jiang, J.-H.; Lu, M.-H.; Chen, Y.-F. Second-order photonic topological insulator with

corner states. Phys. Rev. B: Condens. Matter Mater. Phys. 2018, 98, 205147.

(76) Han, C.; Kang, M.; Jeon, H. Lasing at multidimensional topological states in a two-dimensional photonic crystal structure. ACS Photonics 2020, 7, 2027-2036.

(77) Haus, H. A. Waves and Fieldsin Optoelectronics; Prentice-Hall: Englewood Cliffs, NJ, 1984.

(78) Liu, C.; Di Falco, A.; Molinari, D.; Khan, Y.; Ooi, B. S.; Krauss, T. F.; Fratalocchi, A. Enhanced energy storage in chaotic optical resonators. Nat. Photonics 2013, 7, 473-478.

(79) Yamilov, A.; Cao, H. Highest-quality modes in disordered photonic crystals. Phys. Rev. A: At., Mol., Opt. Phys. 2004, 69, 031803.

(80) Yu, S.; Qiu, C.-W.; Chong, Y.; Torquato, S.; Park, N. Engineered disorder in photonics. Nat. Rev. Mater. 2021, 6, 226-243.

(81) Scheffold, F.; Wiersma, D. Inelastic scattering puts in question recent claims of Anderson localization of light. Nat. Photonics 2013, 7, 934-934.

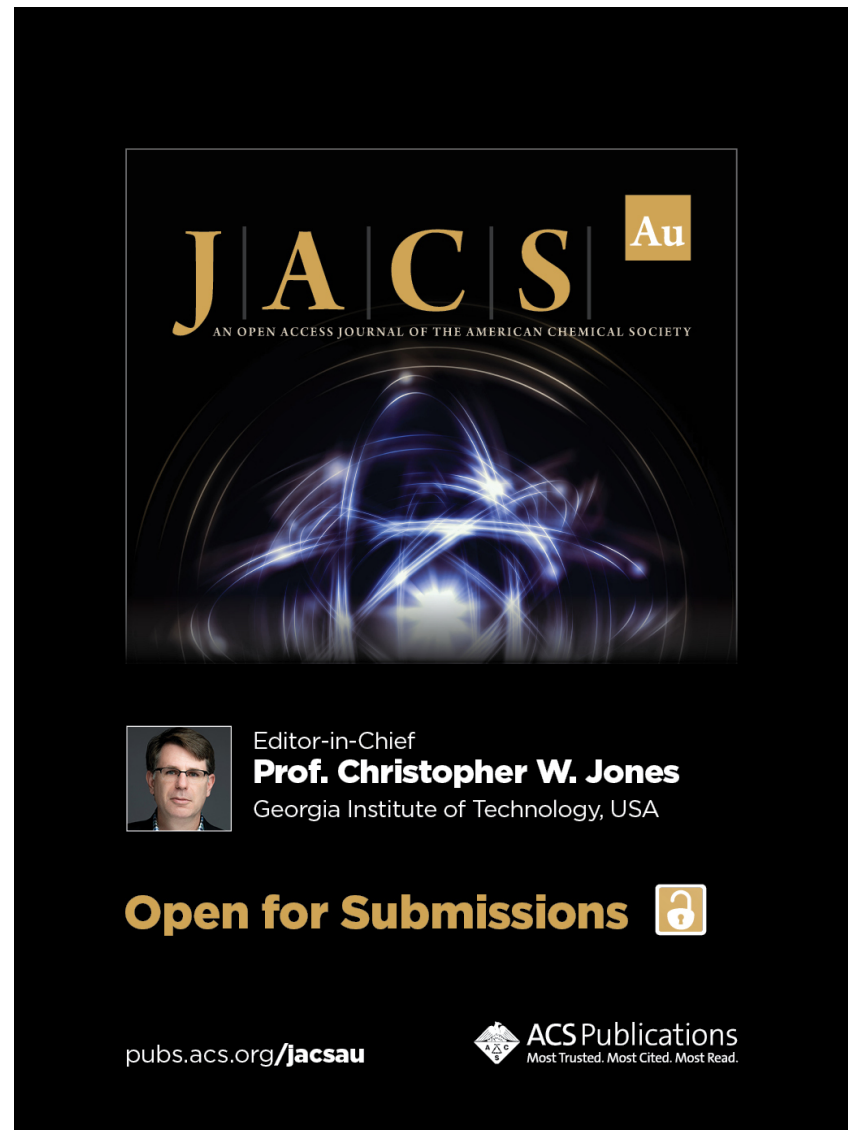

